\title{
Canine scent detection in the diagnosis of lung cancer: revisiting a puzzling phenomenon
}

\author{
R. Ehmann*,\$, E. Boedeker\#,§, U. Friedrich`, J. Sagert", J. Dippon ${ }^{+}$, \\ G. Friedel ${ }^{\#}$ and T. Walles ${ }^{\#}$
}

ABSTRACT: Patient prognosis in lung cancer largely depends on early diagnosis. The exhaled breath of patients may represent the ideal specimen for future lung cancer screening. However, the clinical applicability of current diagnostic sensor technologies based on signal pattern analysis remains incalculable due to their inability to identify a clear target. To test the robustness of the presence of a so far unknown volatile organic compound in the breath of patients with lung cancer, sniffer dogs were applied.

Exhalation samples of $\mathbf{2 2 0}$ volunteers (healthy individuals, confirmed lung cancer or chronic obstructive pulmonary disease (COPD)) were presented to sniffer dogs following a rigid scientific protocol. Patient history, drug administration and clinicopathological data were analysed to identify potential bias or confounders.

Lung cancer was identified with an overall sensitivity of $71 \%$ and a specificity of $93 \%$. Lung cancer detection was independent from COPD and the presence of tobacco smoke and food odours. Logistic regression identified two drugs as potential confounders.

It must be assumed that a robust and specific volatile organic compound (or pattern) is present in the breath of patients with lung cancer. Additional research efforts are required to overcome the current technical limitations of electronic sensor technologies to engineer a clinically applicable screening tool.

KEYWORDS: Biomarker, breath analysis, chronic obstructive pulmonary disease, diagnosis, lung cancer, sniffer dogs

ung cancer continues to represent the second most frequent cancer form in males and females, with 391,000 cases $\cdot \mathrm{yr}^{-1}$ in Europe [1]. Moreover, it is the most common cause of death from cancer, with an estimated 342,000 deaths $\cdot \mathrm{yr}^{-1}$. The prognosis of lung cancer largely depends on disease discovery at an early stage, when the tumour is still localised [2]. Unfortunately, early lung cancer is not associated with symptoms; therefore, detection is often by chance. Clinical practice has shown that the available diagnostic techniques (such as the various imaging technologies or bronchoscopy, including interventional biopsy procedures) have limitations in reliably discriminating between cancer patients and healthy subjects $[3,4]$. No screening method currently exists to test for lung cancer.
Since 1982, research was conducted to develop sensor arrays and pattern recognition technologies, commonly referred to as "electronic noses", which could detect and recognise odours and flavours [5]. It was hypothesised that these devices may be applicable in identifying volatile organic compounds (VOCs) that are linked to cancers in their early stages, thereby making them potential noninvasive and inexpensive diagnostic tools for the medical community [6, 7]. Over the last three decades, "electronic sensing" or "e-sensing" technologies have undergone important developments and are now used to fulfil industrial needs [8]. However, their applicability in a clinical setting is limited due to the fact that patients are required to not smoke and to fast before breath samples can be taken. Other limiting factors are that an optimised
AFFILIATIONS

*Ambulante Pneumologie, Stuttgart, ${ }^{+}$Dept of Mathematics, University of Stuttgart, Stuttgart,

"Dept of General Thoracic Surgery, Schillerhoehe Hospital, Gerlingen, and

"TeamCanin, Loeffingen, Germany ${ }^{\S}$ Both authors contributed equally.

CORRESPONDENCE

T. Walles

Schillerhoehe Hospital Dept of Thoracic Surgery

Solitudestrasse 18

D-70839 Gerlingen Germany

E-mail: Thorsten.Walles@klinikschillerhoehe.de

Received:

March 232011

Accepted after revision: July 052011 First published online: Aug 182011 
sample collection is necessary, the instruments are very sensitive, the sample analysis is of long duration, and there are high risks of signal interference. Interestingly, despite a large body of experimental work, no lung cancer-specific VOCs or VOC patterns have been identified to date [9].

Every now and then, the medical community's attention is drawn to the phenomenon that dogs may detect cancer in patients [10, 11]. Bearing in mind the limitations of the electronic nose, we became interested in this phenomenon. Consequently, we trained four family dogs and designed a prospective, blinded clinical trial to obtain reliable data regarding diagnostic accuracy as well as discriminability of lung cancer from chronic obstructive pulmonary disease (COPD) as a chronic inflammatory condition, which is often associated with the development of lung cancer. Our findings may contribute to the clinical appraisal of breath analysis as a diagnostic approach to identify lung cancer in patients and raise the bar regarding clinical suitability of electronic nose technologies.

\section{METHODS}

\section{Study design}

Hypotheses were tested in a prospective, blinded clinical trial. The study was approved by the Ethics Committee of the University of Tübingen (434/2009BO1; Tübingen, Germany) and the Medical Association of Baden-Württemberg (B-F-2010-004; Germany), and registered at ClinicalTrials.gov (identifier NCT01141842).

\section{Study subjects}

Breath samples from patients with COPD or suspected lung cancer and from healthy individuals were collected from December 2009 to April 2010 at the Schillerhoehe Hospital (Gerlingen, Germany) and the medical practice "Ambulante Pneumologie" (Stuttgart, Germany) after signed consent was obtained. No restrictions were made regarding food ingestion (including tea, coffee and alcohol) and smoking behaviour (no determined smoke-free interval). Additionally, all participants provided their medical history to determine the risk of lung cancer, other cancers and pulmonary disease, and their medication record to control for confounders, and underwent lung function testing to determine the presence of COPD. Inclusion criteria were males and females, aged 18-80 yrs, and signed informed consent. Exclusion criteria were suspected or confirmed malignant disease (other than lung cancer), previous thoracic surgery and any medical intervention at the chest or the airways (for instance thoracocentesis, aspiration biopsy or diagnostic bronchoscopy) within the preceding 14 days. The participants were classified into three groups as follows. Group A: healthy; group B: lung cancer; group C: COPD. A breath sample of all potential patients with lung cancer was obtained at the beginning of their hospital stay and retained. The decision whether a particular patient was assigned to the cancer group B (or was excluded from the study) was made on the basis of the histology of a tumour biopsy or the resected tumour, after meticulous work-up including bronchoscopy and/or surgery (fig. 1). The histological assessment was made by a trained pathologist and the chief of the Dept of Pathology at the Schillerhoehe Hospital.

\section{Breath sample collection}

Cylindrical glass tubes (Gaßner Glastechnik $\mathrm{GmbH}$, Munich, Germany) that could be closed using removable end caps (rubber) were obtained (12 cm length, $2.2 \mathrm{~cm}$ inner diameter; fig. 2a). The lumen of the glass tube was filled with a polypropylene fleece (Asota $\mathrm{GmbH}$, Linz, Austria) that was impregnated with a silicone oil to have either hydrophilic or hydrophobic absorbing properties (CHT R. Beitlich $\mathrm{GmbH}$, Tübingen, Germany). Two tailored straps of the coated fleece (one hydrophilic and one hydrophobic) were loaded into the lumen of the glass tube. Identical test probes that were prepared by the same persons in a standardised procedure were employed at the two collection facilities. Each tube was handled by all individuals involved in the execution of this study to omit unintended "scent labelling". For breath sampling, each participant exhaled five times through the tube, holding it in their bare hands (fig. 2b). The tubes were capped, labelled and stored at room temperature in a light-tight cabinet until testing.

\section{Lung function testing}

Body plethysmography (flow plethysmography) was performed according to established guidelines (CareFusion, Hoechberg, Germany) [12]. The testing was performed in a sitting position and the patient's height and weight were recorded to calculate the reference values. The diagnosis of COPD was made on the basis of medical history and lung function testing according to established guidelines [13].

\section{Dog training}

Dog training and testing was performed in a separate room that was specifically prepared for the study and was not used otherwise. Four family dogs (two German shepherd dogs, one Australian shepherd dog and one Labrador retriever) of both sexes (two females, two males) aged $2.5-3$ yrs were provided by local dog owners and trained by a professional dog trainer following a reward-based approach to indicate breath samples of patients with lung cancer [14]. Dogs were trained to indicate a positive test tube by lying on the floor in front of the tube with the muzzle touching the test tube. During the training, and also later in the testing, every test tube containing a human breath sample was used only once to preclude simple memory recognition of participants' unique odour signatures.

\section{Breath testing}

Three tests were performed in May 2010 to investigate whether the sniffer dogs were able to identify lung cancer among four healthy controls (Test I), to discriminate lung cancer from COPD when tested among four patients with COPD (Test II), and from four representatives of a mixed study population of COPD patients and healthy controls (Test III) (table 1). For testing, the probes were positioned in five separate retainers on the floor, with the rubber caps removed (fig. 2c). Each probe was chosen randomly from the group stack (groups A, B and C). The observers of the dogs' indication were blinded: probe drawing and positioning was concealed from the dogs, their dog handlers, and the study observers by an opaque curtain. In each test, only one probe of a patient with confirmed lung cancer (group B) was used. The position of this probe (retainer 1-5) was determined by throwing a die (fig. $2 \mathrm{~d}$ ), with " 6 " requiring a re-throw. The remaining retainers were randomly filled with test tubes according to test requirements (table 1). For testing, the person who positioned the test tubes left the room, the curtain was opened and the dogs were commanded to sniff the deployed 
a)

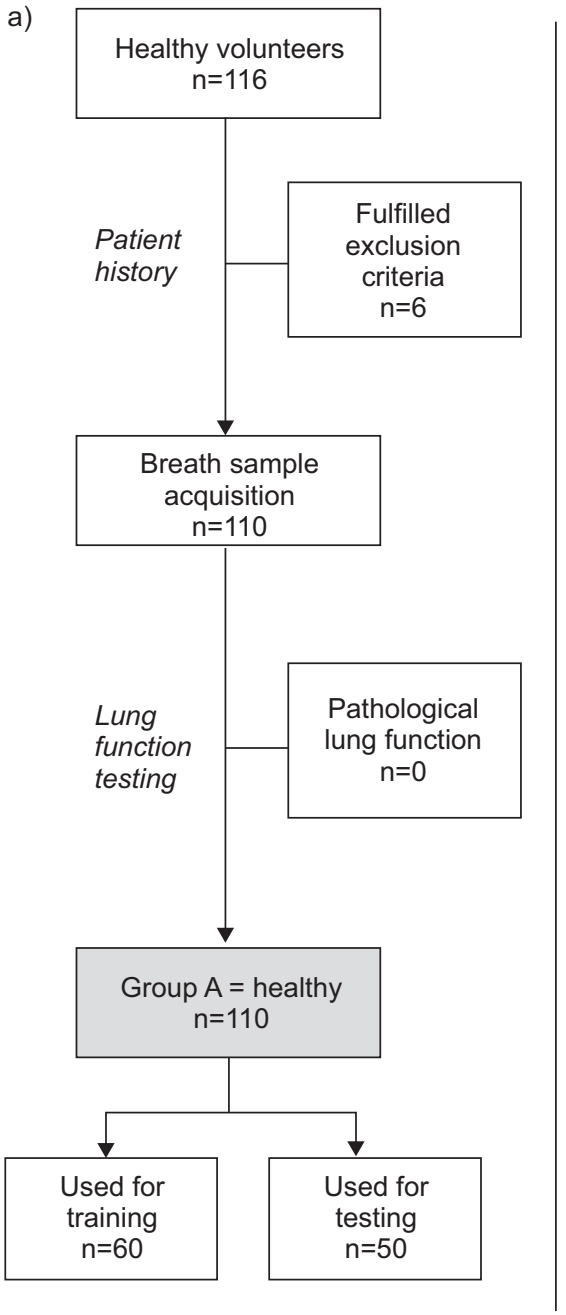

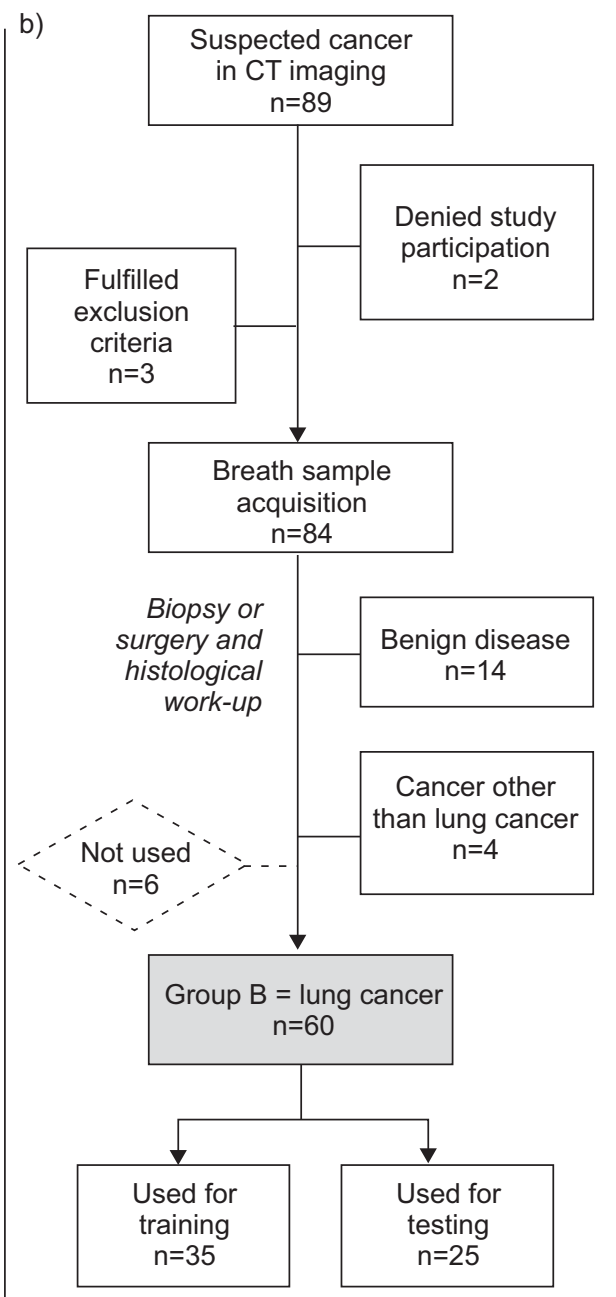

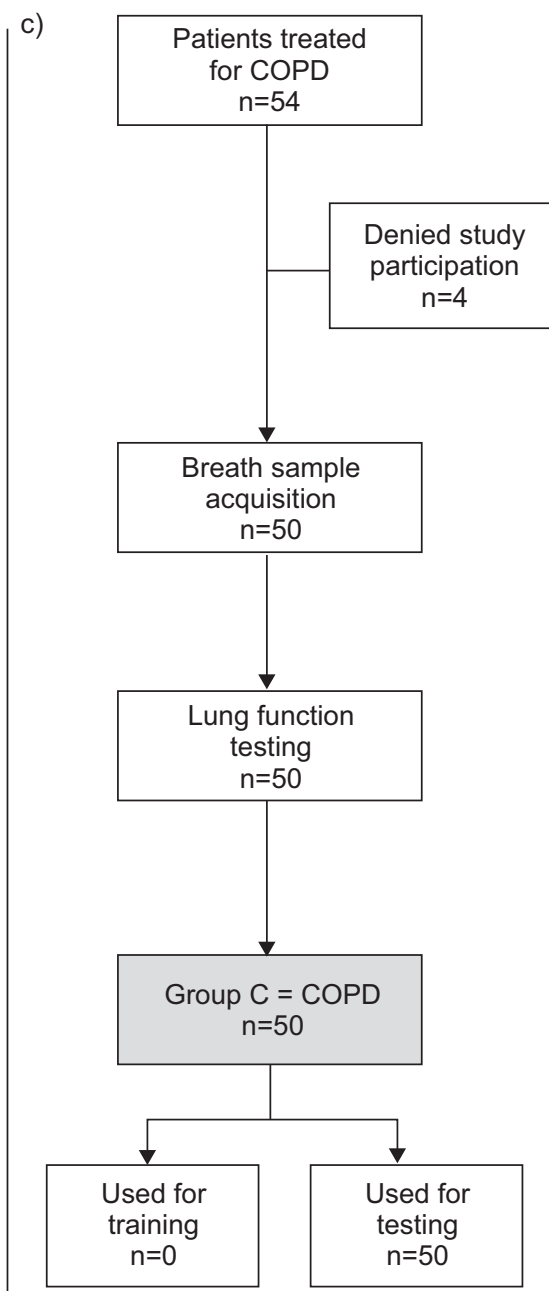

FIGURE 1. Flow diagram showing the criteria for group assignment and reasons for individuals to be excluded. a) Healthy volunteers tested to be included into group $A$. b) Patients suspected of having lung cancer on the basis of patient history and pathological imaging to be included into group B after confirmation of the diagnosis by histology. c) Patients treated for chronic obstructive pulmonary disease (COPD) to be included into group C. The flow chart indicates that breath samples were obtained at the beginning of each individual patient evaluation process and were assigned to the respective study groups thereafter. CT: computed tomography.

probes. Two observers documented the dogs' indications and matched them with the probe array after every test round.

\section{Statistical analysis}

Statistical analysis was performed with the statistical software package $\mathrm{R}$ (version 2.11.0; www.r-project.org) and SPSS (version 15 for Windows; SPSS Inc., Chicago, IL, USA). Fisher's exact test for categorical data was applied to test for homogeneity and compare the relative frequency of events between groups. Groups of continuous data were compared by Wilcoxon's test with continuity correction. The Kruskal-Wallis test was chosen to analyse three groups of continuous data simultaneously. For pairwise comparison of several groups, Holm's method was applied to adjust p-values for multiple testing. Fleiss' Kappa was performed to assess the inter-rater agreement of trained dogs in the experimental setting, with $\kappa=1$ indicating complete rater agreement and $\kappa=0$ indicating agreement only by chance. Mixed effects logistic regression was applied to model the dependence of sample age and medication on the dogs' indication. All p-values were twosided, and a $p$-value of $<0.05$ was considered to indicate statistical significance.

\section{RESULTS}

\section{Composition of training and test groups}

Applying the inclusion and exclusion criteria, 220 participants were enrolled (table 2): 110 healthy individuals (group A), 60 patients with histologically confirmed lung cancer (group B) and 50 with COPD (group C). Differing from our primary inclusion criteria, we had to include patients who had undergone diagnostic bronchoscopy within the last 14 days to facilitate sufficient patient numbers. For dog training, breath samples of 60 healthy volunteers and 35 patients with lung cancer were needed. No training was performed for COPD. To ascertain that breath samples of similar donors were used in dog training and testing, training and test subgroups of groups A and B were compared. Here, no relevant discrepancies were found. In addition, a comparison of the three tested (sub)groups A, B and C revealed 

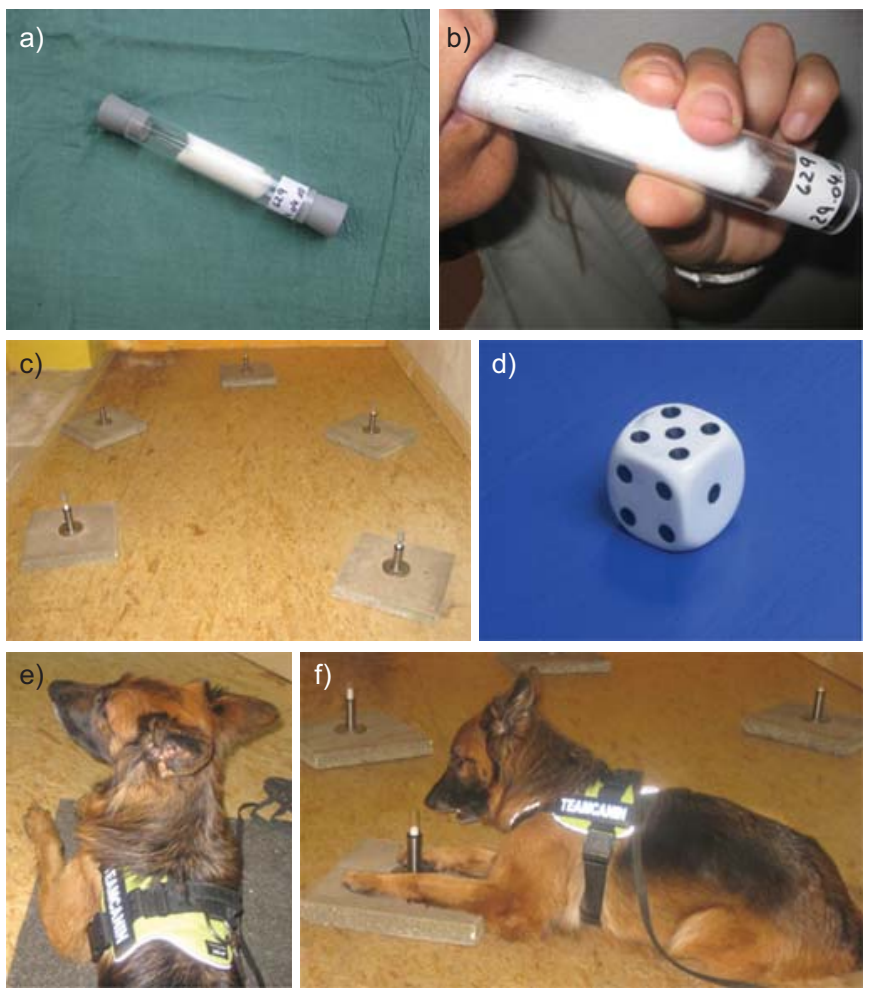

FIGURE 2. Applied methods for breath sampling and testing. a) Glass tube used for breath sampling. The lumen is filled with the polypropylene fleece. b) For breath sampling, study participants exhaled five times through the collection device. c) Test set-up showing the probe retainers. d) The position of the lung cancer samples was randomised. e) Sniffer dogs were trained to identify lung cancer in the breath sample of patients. f) The dogs were trained to indicate a positive test tube by lying on the floor in front of the tube with the muzzle touching the test tube.

no significant statistical difference regarding their constitution. As a consequence of the training strategy (applying breath samples of groups A and B, but not C), the sample age of group C was significantly less.

\section{Lung function in training and test groups}

Lung function parameters were surveyed to determine whether study participants had a normal or limited lung function (table 3) and whether there was a difference between training and test groups. In group A, statistical analysis indicated slightly increased values for the relative vital capacity and forced expiratory volume in $1 \mathrm{~s}$ in the training group. In contrast, all lung function parameters in group $\mathrm{C}$ showed explicit obstructive patterns.

\section{Training and test tumour stages}

A comparison of the underlying tumour stages applied for dog training $(n=35)$ and testing $(n=25)$ showed a shift towards higher tumour stages in the test group (fig. 3a). However, a considerable number of employed breath samples were obtained from patients with early and locally advanced lung cancer disease of Union for International Cancer Control (UICC) stage I to IIIa (46\% in the training and $36 \%$ in the test subgroup). Regarding tumour histology, the majority of tested breath samples in group B was obtained from patients with adenomatous nonsmall cell lung
TABLE 1 Breath sample distribution throughout the study

\begin{tabular}{lccc} 
& $\begin{array}{c}\text { Group A } \\
\text { healthy }\end{array}$ & $\begin{array}{c}\text { Group B } \\
\text { lung cancer }\end{array}$ & $\begin{array}{c}\text { Group C } \\
\text { COPD }\end{array}$ \\
\hline $\begin{array}{l}\text { Training } \\
\text { Test I }\end{array}$ & 60 & 35 & \\
Test II & 40 & 10 & \\
Test III & 10 & 10 & 40 \\
Total & 110 & 5 & 10 \\
\hline
\end{tabular}

Data are presented as n. COPD: chronic obstructive pulmonary disease.

cancer (fig. 3b). Diagnostic work-up following breath sampling identified four tumours as small cell lung cancers.

\section{Lung cancer identification by sniffer dogs}

Tests I to III were performed within 2 days with no modifications in the test protocol. Following the search command, each investigational course (for the five assembled breath samples) took $<15 \mathrm{~s}$. Every dog indication had to be definite and hesitation free with the dog lying in front of the test tube. If it was not, the indication was assessed as incorrect. Two blinded study observers recorded the dogs' indications (table 4). There were no disagreements in the two observers' records. The hit ratio for the four individual dogs was different throughout the course of experiments, ranging from $68 \%$ to $84 \%$ (table 5). The accuracy of the dog's indication did not favour advanced tumour stages and was $100 \%$ for UICC stage I, 75\% for UICC stages IIa and Ilb, $94 \%$ for UICC stage IIIa, 75\% for UICC stage IIIlb and 63\% for UICC stage IV. The overall sensitivity was $71 \%$ and the specificity was $93 \%$, with the positive and negative predictive values being $72 \%$ and $93 \%$, respectively (table 4 ). The inter-rater variability of the four dogs was moderate $(\kappa=0.436$; table 5$)$. The best results were obtained in test III (mixed population), the worse in test I (lung cancer versus healthy controls). Therefore, we defined the "corporate dog decision", which requires at least three dogs making the same decision. The corporate dog decision analysis, however, did not ameliorate our test scores, resulting in a sensitivity of $72 \%$, a specificity of $94 \%$ and positive and negative predictive values of $75 \%$ and $93 \%$, respectively.

\section{Controlling for confounders}

The distribution of active smokers was similar between groups (table 2). The inclusion of patients who had previously undergone diagnostic bronchoscopy did not influence the sample classification by the dogs $(\mathrm{p}=0.6729, \mathrm{CI} 0.1618-12.2953$, odd ratio 1.4751). To eliminate potential bias and confounders, we obtained a detailed medical history and documented all drugs taken by the study participants. Collectively, we recorded 22 diseases and 112 drugs. In our statistical analysis, an inhomogeneous distribution emerged for four diseases and 20 active agents (see online supplementary material table 6). Subsequent mixed effect logistic regression, however, identified nine potential confounders (see online supplementary material table 7). Also, employing logistic regression, we excluded the eventuality that the difference of sample age (at time of testing) and age of participants had an influence on the dogs' sample classification (see online supplementary material fig. 4). 
TABLE 2 Composition of training and test groups

\begin{tabular}{|c|c|c|c|c|c|c|c|c|c|c|}
\hline & \multicolumn{4}{|c|}{ Group A: healthy } & \multicolumn{4}{|c|}{ Group B: lung cancer } & \multicolumn{2}{|c|}{ Group C: COPD } \\
\hline & All & Training & Test & p-value ${ }^{\#}$ & All & Training & Test & p-value ${ }^{\#}$ & Test & p-value \\
\hline Subjects & 110 & 60 & 50 & & 60 & 35 & 25 & & 50 & \\
\hline Age yrs & $46.2 \pm 14.0$ & $45.7 \pm 12.5$ & $46.8 \pm 15.8$ & NS & $63.6 \pm 10.3$ & $65.3 \pm 9.8$ & $62.6 \pm 11.1$ & NS & $66.7 \pm 6.6$ & $<0.001$ \\
\hline Sex M/F & $26 / 74$ & 28/72 & $24 / 76$ & & $78 / 22$ & $71 / 29$ & $88 / 12$ & & $56 / 44$ & \\
\hline $\begin{array}{c}\text { History of } \\
\text { cancer }\end{array}$ & $0(0)$ & $0(0)$ & $0(0)$ & & $0(0)$ & $0(0)$ & $0(0)$ & & $3(5)$ & \\
\hline $\begin{array}{l}\text { Previous } \\
\text { surgery }\end{array}$ & $0(0)$ & $0(0)$ & $0(0)$ & & $0(0)$ & $0(0)$ & $0(0)$ & & $1(1.7)$ & \\
\hline $\begin{array}{l}\text { Previous } \\
\text { intervention }\end{array}$ & $0(0)$ & $0(0)$ & $0(0)$ & & $34(57)$ & $18(51)$ & $16(64)$ & & $0(0)$ & $<0.001$ \\
\hline
\end{tabular}

Data are presented as $n$, mean \pm SD, \%/\% or $n(\%)$, unless otherwise stated. COPD: chronic obstructive pulmonary disease; M: male; F: female; BMI: body mass index;

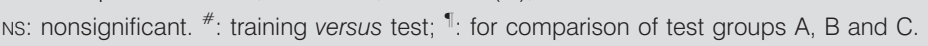

\section{DISCUSSION}

This meticulous characterisation of 125 breath samples tested by four sniffer dogs confirms the existence of a stable marker (or scent pattern) that is strongly associated with lung cancer and independent from COPD, but can be reliably discriminated from tobacco smoke, food odours and (potential) drug metabolites.

Since their first delineation by PAULING et al. [15] in 1971, 3,481 different VOCs have been described in the human breath, most of them in picomolar concentrations $\left(10-12 \mathrm{~mol} \cdot \mathrm{L}^{-1}\right.$ or particles per trillion) [15, 16]. It has been hypothesised that tumours produce VOCs; therefore, breath analysis might be a very sensitive and, at the same time, noninvasive method to screen for or diagnose cancer. In particular, this is interesting for lung cancer due to its site of origin, prevalence in industrialised societies and unfavourable prognosis. However, the metabolic origin of tumour-associated VOCs remains speculative [17] Nonetheless, three recent publications have demonstrated that breath samples from patients with lung cancer and those from healthy subjects can be distinguished by electronic nose technology [18-20]. Tumour stage did not influence the outcome in any of the studies, implying that exhaled breath profiling has the potential to evolve as a screening test for lung cancer, once specific markers have been identified [6].

TABLE 3 Lung function tests in training and test groups

\begin{tabular}{|c|c|c|c|c|c|c|c|c|c|c|}
\hline & \multicolumn{4}{|c|}{ Group A: healthy } & \multicolumn{4}{|c|}{ Group B: lung cancer } & \multicolumn{2}{|c|}{ Group C: COPD } \\
\hline & All & Training & Test & p-value ${ }^{\#}$ & All & Training & Test & p-value ${ }^{\#}$ & Test & p-value \\
\hline Subjects & 110 & 60 & 50 & & 60 & 35 & 25 & & 50 & \\
\hline VC L & $4.0 \pm 1.0$ & $4.1 \pm 1.0$ & $3.8 \pm 0.8$ & NS & $3.5 \pm 1.1$ & $2.9 \pm 1.1$ & $3.5 \pm 1.1$ & NS & $2.8 \pm 0.8$ & $<0.001$ \\
\hline VC \% & $107 \pm 14$ & $111 \pm 12$ & $103 \pm 16$ & $<0.01$ & $83 \pm 21$ & $79 \pm 22$ & $83 \pm 21$ & & $79 \pm 20$ & \\
\hline FEV 1 L & $3.3 \pm 0.8$ & $3.4 \pm 0.8$ & $3.2 \pm 0.8$ & NS & $2.4 \pm 0.8$ & $2.1 \pm 1.0$ & $2.4 \pm 0.8$ & & $1.6 \pm 0.6$ & \\
\hline FEV $_{1} \%$ & $109 \pm 15$ & $112 \pm 13$ & $105 \pm 17$ & $<0.05$ & $74 \pm 25$ & $73 \pm 25$ & $76 \pm 25$ & & $61 \pm 19$ & \\
\hline $\mathrm{FEV}_{1} \% \mathrm{VC}$ & $81.2 \pm 5.9$ & $80.6 \pm 5.9$ & $82.0 \pm 5.9$ & NS & $65 \pm 13$ & $65 \pm 13$ & $66 \pm 12$ & & $59 \pm 11$ & \\
\hline TLC L & $6.0 \pm 1.3$ & $5.9 \pm 1.3$ & $6.0 \pm 1.3$ & & $5.8 \pm 1.3$ & $5.9 \pm 1.3$ & $5.8 \pm 1.3$ & & $7.1 \pm 1.4$ & \\
\hline TLC \% & $105 \pm 18$ & $104 \pm 13$ & $107 \pm 22$ & & $92 \pm 20$ & $97 \pm 21$ & $85 \pm 16$ & & $120 \pm 20$ & \\
\hline RV L & $1.8 \pm 1.1$ & $1.6 \pm 0.8$ & $2.1 \pm 1.3$ & & $2.5 \pm 1.2$ & $2.7 \pm 1.4$ & $2.1 \pm 0.7$ & & $4.4 \pm 1.2$ & \\
\hline RV \% & $97 \pm 52$ & $87 \pm 30$ & $109 \pm 69$ & & $106 \pm 49$ & $118 \pm 58$ & $88 \pm 25$ & & $187 \pm 56$ & \\
\hline GOLD & $0 \pm 0.2$ & $0 \pm 0.2$ & $0 \pm 0.3$ & & $1.1 \pm 1.3$ & $1.2 \pm 1.4$ & $1.1 \pm 1.3$ & & $1.9 \pm 1.0$ & \\
\hline
\end{tabular}

Data are presented as $n$ or mean $\pm \mathrm{SD}$, unless otherwise stated. COPD: chronic obstructive pulmonary disease; VC: vital capacity; FEV 1 : forced expiratory volume in 1 s; TLC: total lung capacity; RV: residual lung volume; GOLD: stage of COPD according to the Global Initiative for Chronic Obstructive Lung Disease; NS: nonsignificant.

\# : training versus test; ${ }^{\bullet}$ : for comparison of test groups $A, B$ and $C$. 

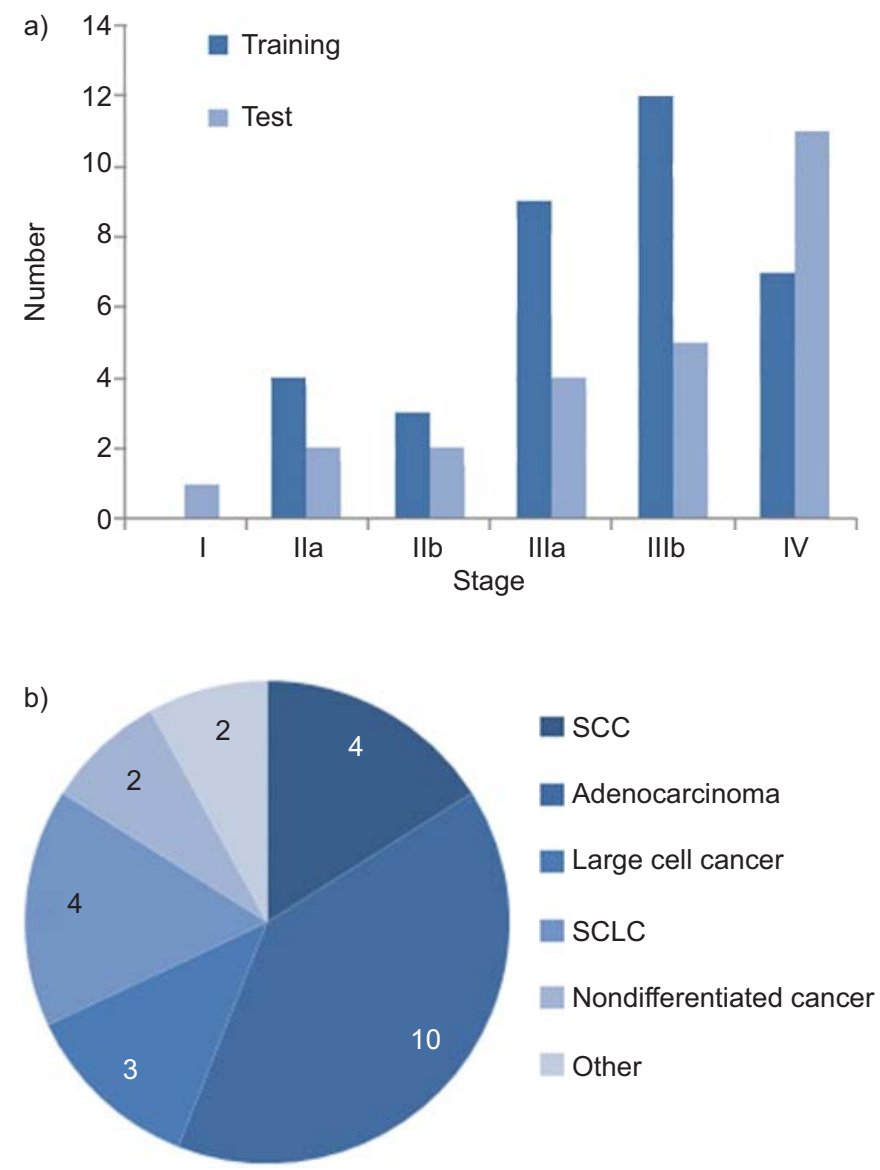

FIGURE 3. Itemisation of trained and tested tumour stages. a) Early and advanced lung cancer tumour stages were trained and tested. b) Distribution of tested tumour histologies. SCC: squamous cell carcinoma; SCLC: small cell lung cancer.

COPD often precedes and accompanies lung cancer in smoking patients [21]. COPD is characterised by typical lung function deterioration, chronic systemic and local airway inflammation and structural changes in lung parenchyma. It has been shown that the level of exhaled biomarkers is altered in patients with COPD compared with healthy control subjects $[9,20]$. Moreover, since the development of lung cancer is much more frequent in COPD patients than in healthy controls, attention needs to be focused on the subtle differences in exhaled biomarker profiles between lung cancer and COPD [6].

\section{TABLE 5 Hit ratio of sniffer dogs}

\begin{tabular}{|c|c|c|c|c|c|}
\hline & Dog 1 & Dog 2 & Dog 3 & Dog 4 & $\begin{array}{l}\text { Corporate } \\
\text { decision }{ }^{\#}\end{array}$ \\
\hline \multicolumn{6}{|l|}{ Test I } \\
\hline Correct & 7 & 4 & 6 & 5 & \\
\hline False & 3 & 6 & 4 & 5 & \\
\hline \multicolumn{6}{|l|}{ Test II } \\
\hline Correct & 9 & 8 & 7 & 8 & \\
\hline False & 1 & 2 & 3 & 2 & \\
\hline \multicolumn{6}{|l|}{ Test III } \\
\hline Correct & 5 & 5 & 4 & 5 & \\
\hline False & 0 & 0 & 1 & 0 & \\
\hline \multicolumn{6}{|l|}{ Overall } \\
\hline Correct & 21 & 17 & 17 & 18 & \\
\hline False & 4 & 8 & 8 & 7 & \\
\hline Percentage correct & 84 & 68 & 68 & 72 & \\
\hline Inter-ratervariability $к$ & \multicolumn{4}{|c|}{0.436} & \\
\hline Sensitivity & & & & & $0.72(0.51-0.88)$ \\
\hline Specificity & & & & & $0.94(0.87-0.98)$ \\
\hline PPV & & & & & $0.75(0.53-0.91)$ \\
\hline NPV & & & & & $0.93(0.86-0.97)$ \\
\hline
\end{tabular}

Data are presented as $\mathrm{n}$ or value (95\% confidence interval), unless otherwise stated. PPV: positive predictive value; NPV: negative predictive value. ${ }^{*}$ : at least three dogs alike.

Research on electronic nose technologies continues to advance and optimise its technical capabilities [6, 9]. However, the currently required breath sampling procedures are very complex, and their analysis is interference prone. Sample analysis requires $10 \mathrm{~min}$ at best and the obtained detection rates vary considerably [6]. Therefore, it is currently difficult to predict when a clinically applicable diagnostic device for breath analysis will be available.

In contrast, and virtually on the verge of respectability, sniffer dogs emerge as "detection devices" in the medical literature. Initial interest in dogs being able to detect cancer in humans developed when WiLLIAMS and PемвROKE [10] sent a letter to The Lancet in 1989 where they described a case where a female was encouraged to get a skin lesion examination because of her dog's inordinate amount of interest in the spot on her skin. The outstanding sensitivity of the canine olfactory system has been acknowledged by using sniffer dogs in military and civilian

TABLE 4 Cross-tabulation of the dogs' indication and the presence of cancer

Breath sample of volunteer without cancer (groups $\mathrm{A}+\mathrm{C}$ )
Breath sample of volunteer with confirmed lung cancer (group B)
Total
Dogs indicating absence of lung cance

Total

$\begin{array}{ccc}28 & 71 & 99 \\ 372 & 29 & 401 \\ 400 & 100 & 500\end{array}$


service for detection of a variety of odours. Moreover, sniffer dogs have been employed in pre-clinical studies for cancer diagnosis [22, 23]. In 2006, McCuLLOCH et al. [14] reported a sensitivity and specificity of $99 \%$ for sniffer dogs to diagnose lung cancer from patients' breath samples. However, this study might have been biased by odours related to other diseases, therapies and smoking. In the present study, trained sniffer dogs were able to identify lung cancer in one out of five probes with an overall sensitivity of $71 \%$ and specificity of $93 \%$.

In great contrast to related previous studies, the focus of the present work was to exclude potential confounders and bias. No relevant differences were identified between training and test groups (tables 2 and 3). Although the study groups showed an inhomogenous distribution regarding age, sex and breath sample age (table 2), multivariate analysis excluded an association between these characteristics and the dogs' indication. The lung function parameters were pathological for group C, as was hypothesised by the applied study protocol (table 3). The 6month training period resulted in a Fleiss' Kappa value of $44 \%$, indicating moderate rater variability between dogs. Since an improvement of lung cancer identification capabilities can be identified along the test series (table 5), an ongoing training effect must be assumed, calling for even longer dog training in future studies. In theory, breath sample recovery from the study participants without using gloves might have introduced confounding odours. This leaves open the possibility that cancer-associated odours may be emitted through the skin, as well as exhaled during respiration. However, this does not help to explain how the dogs were able to differentiate between groups $\mathrm{A}, \mathrm{B}$ and $\mathrm{C}$.

Mixed effect logistic regression analysis identified nine potential confounders among 112 drugs (see online supplementary material table 7). Therefore, methoclopramide, enoxaparin, dihydrocodein, triotropiumbromide, clopidogrel, ezetmib, marcumar, verapamil and metoprolol may be potential confounders. Actually, the first three of these drugs have been administered to in-patients diagnosed for lung cancer and, therefore, may represent a study bias. In contrast, metropolol, verapamil and tiotropiumbromide were consistently distributed between lung cancer and COPD patients, but not found in healthy volunteers. Marcumar, clopidogrel and ezetimib were present exclusively in COPD patients. The tested tumour histologies represent the ordinary clinical distribution, and the included tumour stages reflect the entire spectrum of disease (fig. 3). Although the data indicate that sniffer dogs can identify early-stage lung cancer, their foundation is too small to conclude that sniffer dogs may be applicable for reliable lung cancer screening. Interestingly, advanced tumour UICC stage IV may impair the display accuracy of sniffer dogs. Here, the presence of secondary lung tissue reactions (e.g. inflammation or necrosis) may be imputed.

Exhaled breath analysis is a promising approach towards future noninvasive lung cancer screening methods. The final goal of our investigation is the development of a clinically applicable screening test for detection of lung cancer. Using sniffer dogs as a detection device, our results set a benchmark for the identification of lung cancer in the breath samples of patients and the discrimination of lung cancer and underlying COPD. Here, we confirm the presence of a detectable marker in the breath of patients, which is strongly associated with lung cancer but independent from COPD. However, this marker or pattern, despite being reliably detectable even in the presence of tobacco smoke, food odours and drug metabolites, is still unknown. In order to proceed towards noninvasive lung cancer screening methods, precise identification of compounds observed in exhaled breath of lung cancer patients is desirable. Acknowledging the complexity of this endeavour, the integration of sniffer dogs into research strategies may be useful. In our study, sniffer dogs reliably identified lung cancer, whereas electronic nose technologies detect thousands of scents, of which the majority is, to date, not specifiable. Unfortunately, dogs cannot communicate the biochemistry of the scent of cancer.

\section{SUPPORT STATEMENT}

The study was funded by private financial resources of R. Ehmann, E. Boedeker and T. Walles.

\section{CLINICAL TRIAL}

This study is registered at ClinicalTrials.gov with identifier NCT01141842.

\section{STATEMENT OF INTEREST}

Statements of interest for R. Ehmann, E. Boedeker, U. Friedrich $\mathrm{T}$. Walles and the study itself can be found at www.erj.ersjournals. $\mathrm{com} / \mathrm{site} / \mathrm{misc} /$ statements.xhtml

\section{ACKNOWLEDGEMENTS}

S. Layland (Fraunhofer IGB, Stuttgart, Germany) edited the manuscript for language and grammar. The authors thank G. Oster and F. Baur (TeamCanin, Loeffingen, Germany) for their dedication and making their dogs available for the training, as well as G. Günther (TeamCanin) and A. Hofbauer (Schillerhoehe Hospital, Gerlingen, Germany) for their back office. The authors are indebted to the doctors and doctor's assistants of the Ambulante Pneumologie (Stuttgart, Germany) for their support in patient recruitment and sample collection. This study would not have been possible without the docility and excitement of the dogs, "Kessie", "Benny", "Hector" and "Bonnie".

\section{REFERENCES}

1 Ferlay J, Parkin DM, Steliarova-Foucher E. Estimates of cancer incidence and mortality in Europe in 2008. Eur J Cancer 2010; 46: 765-781.

2 Cataldo JK, Dubey S, Prochaska JJ. Smoking cessation: an integral part of lung cancer treatment. Oncology 2010; 78: 289-301.

3 Hinterthaner M, Stamatis G. Heutige Rolle der Mediastinoskopie und der Remediastinoskopie [Role of mediastinoscopy and repeat mediastinoscopy today]. Chirurg 2008; 79: 38-44.

4 Schöder H, Gönen M. Screening for cancer with PET and PET/CT: potential and limitations. I Nucl Med 2007; 48: Suppl. 1, 4S-18S.

5 Persaud K, Dodd G. Analysis of discrimination mechanisms in the mammalian olfactory system using a model nose. Nature 1982; 299: 352-355.

6 Horváth I, Lázár Z, Gyulai N, et al. Exhaled biomarkers in lung cancer. Eur Respir J 2009; 34: 261-275.

7 Peng G, Hakim M, Broza YY, et al. Detection of lung, breast, colorectal, and prostate cancers from exhaled breath using a single array of nanosensors. Br J Cancer 2010; 103: 542-551.

8 Peris M, Escuder-Gilabert L. A 21st century technique for food control: electronic noses. Anal Chim Acta 2009; 638: 1-15.

9 D'Amico A, Pennazza G, Santonico M, et al. An investigation on electronic nose diagnosis of lung cancer. Lung Cancer 2010; 68: 170-176.

10 Williams H, Pembroke A. Sniffer dogs in the melanoma clinic? Lancet 1989; 1: 734. 
11 Church J, Williams H. Another sniffer dog for the clinic? Lancet 2001; 358: 930.

12 Wanger J, Clausen JL, Coates A, et al. Standardisation of the measurement of lung volumes. Eur Respir J 2005; 26: 511-522.

13 Rabe KF, Hurd S, Anzueto A, et al. Global strategy for the diagnosis, management, and prevention of chronic obstructive pulmonary disease: GOLD executive summary. Am J Respir Crit Care Med 2007; 176: 532-555.

14 McCulloch M, Jezierski T, Broffman M, et al. Diagnostic accuracy of canine scent detection in early- and late-stage lung and breast cancers. Integr Cancer Ther 2006; 5: 30-39.

15 Pauling L, Robinson AB, Teranishi R, et al. Quantitative analysis of urine vapor and breath by gas-liquid partition chromatography. Proc Natl Acad Sci USA 1971; 68: 2374-2376.

16 Phillips M, Herrera J, Krishnan S, et al. Variation in volatile organic compounds in the breath of normal humans. J Chromatogr B Biomed Sci Appl 1999; 729: 75-88.
17 Sponring A, Filipiak W, Mikoviny T, et al. Release of volatile organic compounds from the lung cancer cell line NCI-H2087 in vitro. Anticancer Res 2009; 29: 419-426.

18 Machado RF, Laskowski D, Deffenderfer O, et al. Detection of lung cancer by sensor array analyses of exhaled breath. Am J Respir Crit Care Med 2005; 171: 1286-1291.

19 Di Natale C, Macagnano A, Martinelli E, et al. Lung cancer identification by the analysis of breath by means of an array of non-selective gas sensors. Biosens Bioelectron 2003; 18: 1209-1218.

20 Dragonieri S, Annema JT, Schot R, et al. An electronic nose in the discrimination of patients with non-small cell lung cancer and COPD. Lung Cancer 2009; 64: 166-170.

21 Yao H, Rahman I. Current concepts on the role of inflammation in COPD and lung cancer. Curr Opin Pharmacol 2009; 9: 375-383.

22 Gordon RT, Schatz CB, Myers LJ, et al. The use of canines in the detection of human cancers. J Altern Complement Med 2008; 14: 61-67.

23 Moser E, McCulloch M. Canine scent detection of human cancers: a review of methods and accuracy. J Vet Behav 2010; 5: 145-152. 